Published in final edited form as:

Eur J Neurosci. 2013 October ; 38(7): 3044-3053. doi:10.1111/ejn.12309.

\title{
The complex relationship between the light-entrainable and methamphetamine-sensitive circadian oscillators: Evidence from behavioral studies of Period-mutant mice
}

\author{
Julie S. Pendergast ${ }^{1}$, Kevin D. Niswender ${ }^{2}$, and Shin Yamazaki ${ }^{1,{ }^{*}}$ \\ ${ }^{1}$ Department of Biological Sciences, Vanderbilt University, Nashville, TN, 37235 \\ ${ }^{2}$ VA Tennessee Valley Healthcare System, Nashville, TN 37212 and Division of Diabetes, \\ Endocrinology \& Metabolism, Department of Medicine, Vanderbilt University School of Medicine, \\ Nashville, TN 37232
}

\section{Abstract}

The methamphetamine-sensitive circadian oscillator (MASCO) is an enigmatic circadian clock whose output is observed during continuous consumption of low-dose methamphetamine. The MASCO rhythm persists when the light-entrainable pacemaker in the suprachiasmatic nucleus (SCN) is lesioned, but the anatomical location of MASCO is unknown. We recently found that the period of the MASCO rhythm is unusually short $(21 \mathrm{~h})$ in mice with disruption of all three paralogs of the canonical clock gene, Period. In this study, we investigated the contribution of each Period paralog to timekeeping in MASCO. We measured wheel-running activity rhythms in intact and SCN-lesioned Perl-, 2- and 3-mutant mice administered methamphetamine, and found that none of the mice displayed a short (21-h) period, demonstrating that no single Period gene is responsible for the short-period MASCO rhythm of $\mathrm{Perl}^{-/-} / \mathrm{Per}^{-/-} / \mathrm{Per}^{-/-}$mice. We also found that the periods of activity rhythms in constant darkness were lengthened by methamphetamine treatment in intact wild-type, $\mathrm{Perl}^{-/-}$and $\mathrm{Per}^{-/-}$mice but not $\mathrm{Per}^{-/-}$mice, and $P e r 2^{-/-}$mice had two distinct activity rhythms upon release to constant light. These data suggest that the SCN and MASCO are not coupled in $P e r 2^{-1-}$ mice. The MASCO rhythm in $\mathrm{Perl}^{-/-} / \mathrm{Per}^{-/-}$mice in constant darkness alternated between a short (22-h) and a long (27-h) period. This pattern could result from two coupled oscillators that are not synchronized to each other, or from a single oscillator displaying birhythmicity. Finally, we propose a working model of the in vivo relationship between MASCO and the SCN that poses testable hypotheses for future studies.

\section{Keywords}

suprachiasmatic nucleus; MASCO; limit cycle; birhythmicity; coupling

\footnotetext{
*To whom correspondence should be addressed: Shin Yamazaki (present address), Department of Neuroscience, University of Texas Southwestern Medical Center, 5323 Harry Hines Blvd, Dallas, TX 75390-9111, Fax: 214-648-1801,

shin.yamazaki@utsouthwestern.edu.

J. S. Pendergast's present address: Division of Diabetes, Endocrinology \& Metabolism, Department of Medicine, Vanderbilt

University School of Medicine, Nashville, TN 37232

The authors declare no competing financial or non-financial interests.
} 


\section{Introduction}

Circadian rhythms are self-sustained oscillations in physiology and behavior with endogenous periods of $\sim 24 \mathrm{~h}$ that can be entrained to environmental cues such as the lightdark cycle and food availability (Takahashi et al., 2001). The mammalian circadian system is organized hierarchically, such that the master light-entrainable circadian clock in the suprachiasmatic nucleus ( $\mathrm{SCN}$ ) of the anterior hypothalamus coordinates the phases of oscillators in peripheral tissues (Yamazaki et al., 2000; Yoo et al., 2004). Behavioral experiments have revealed the existence of two enigmatic oscillators, the methamphetaminesensitive circadian oscillator (MASCO) and the food-entrainable oscillator (FEO), whose overt circadian activity rhythms are revealed by methamphetamine and food restriction (followed by food deprivation), respectively (Honma et al., 1986; Mistlberger, 1994; Tataroglu et al., 2006). Both MASCO- and FEO-controlled activity rhythms persist after SCN lesion, but the anatomical loci of these oscillators are unknown (Honma et al., 1987; Mistlberger, 1994; Davidson, 2009). Indeed, it is possible that they are the same circadian oscillator. Like the SCN, the MASCO and FEO can coordinate the phases of peripheral oscillators (Pezuk et al., 2010), suggesting that they function at the top of the hierarchy of the mammalian circadian system.

As the location of MASCO is unknown, recent studies have focused on the molecular timekeeping mechanism of this oscillator. The primary conclusion from these studies was that the MASCO is a 'non-canonical' circadian oscillator because free-running MASCO rhythms persist when the canonical circadian genes are non-functional (in $\mathrm{Cry} \mathrm{I}^{-/-} / \mathrm{Cry} 2^{-/-}$, $\mathrm{Bmall}^{-/-}$and $\mathrm{Perl}^{-/-} / \mathrm{Per}^{-/-}$mice) even though those mutations render SCN-driven locomotor activity arrhythmic (Honma et al., 2008; Mohawk et al., 2009). In contrast, we recently found that the MASCO rhythm is unusually short $(\sim 21 \mathrm{~h})$ in mice with disruption of all three paralogs of the canonical clock gene Period (Pendergast et al., 2012). These data demonstrate that the Period genes are involved in the timekeeping mechanism in MASCO, but they are not necessary to sustain the oscillation. In the current study, we investigated the contribution of each of the three Period paralogs to timekeeping in MASCO. Our data demonstrate that no single Period gene is responsible for the short-period MASCO rhythm of $\mathrm{Perl}^{-/-} / \mathrm{Per}^{-/-} / \mathrm{Per}^{-/-}$mice. However, during the course of our experiments we identified several intriguing properties of MASCO and its relationship with the SCN. From the data described herein, we propose a working model of the in vivo relationship between the MASCO and SCN.

\section{Materials and methods}

\section{Animals}

We obtained $\mathrm{mPer}^{-/-}, \mathrm{mPer}^{-/-}$and $\mathrm{mPer}^{-/-}$mice (Shearman et al., 2000; Bae et al., 2001) (provided by Dr David Weaver, University of Massachusetts Medical School; congenic with the 129/sv genetic background) and backcrossed them with wild-type C57BL/6J mice (Jackson Laboratory, Bar Harbor, ME, USA) for at least 15 generations (Pendergast et al., 2009;, 2010a; C57BL/6J Perl ${ }^{-/-}$, Per2 ${ }^{-/-}$and Per $^{-/-}$mice are available from The Jackson Laboratory, stock numbers 10491, 10492 and 10493, respectively). Wild-type and Per-mutant mice were generated from intercrossing Per-mutant 
mice. Adult male and female mice (6-39 weeks old) were used for all experiments. Genotyping for the Per genes was performed as previously described (Shearman et al., 2000; Bae et al., 2001). The mice were bred and group-housed in the Vanderbilt University animal facility in a 12-h light-12-h dark cycle[A1] (light intensity 350 lux) and provided food and water ad libitum. All experiments were carried out in accordance with the National Institutes of Health Guidelines regarding the care and use of animals for experimental procedures and were approved by the Institutional Animal Care and Use Committee at Vanderbilt University (M/08/096).

\section{Recording of circadian behavior and methamphetamine treatment}

Animals were singly housed in cages (length $\times$ height $\times$ width: $33 \times 17 \times 14 \mathrm{~cm}$ ) with unlimited access to a running wheel (diameter $11 \mathrm{~cm}$ ), food and water. The cages were placed in light-tight ventilated boxes in light-dark conditions (light intensity, 200-300 lux), in constant darkness (DD)[A2] or in constant light (LL; light intensity, 200-300 lux), as indicated for each experiment. Mice were provided with $0.005 \%$ methamphetamine hydrochloride (Sigma) in their drinking (tap) water as indicated. Cages were changed every 3 weeks. Wheel-running activity (recorded every minute by computer) was monitored and analyzed using ClockLab (Actimetrics, Wilmette, IL, USA). Activity data were doubleplotted in actograms in 5-min bins using the normalized format in ClockLab.

\section{SCN lesions}

All surgery was performed under anesthesia by intraperitoneal administration of ketamine (100mg/kg; Putney Inc., Portland, ME, USA) and xylazine $(10 \mathrm{mg} / \mathrm{kg}$; Lloyd Laboratories, Inc., Shenandoah, IA, USA).[A3] Buprenex $(0.05 \mathrm{mg} / \mathrm{kg}$; Reckitt Benckiser Pharmaceuticals Inc., Richmond, VA, USA) was administered subcutaneously immediately after surgery. Mice were placed in a stereotaxic apparatus (Stoelting, Wood Dale, IL, USA) and bilateral lesions were made by passing a 1.1-mA direct[A4] current through a teflon-coated tungsten electrode (Unique Medical, Osaka, Japan, or A-M systems, catalog number 575500, Sequim, WA, USA) for $20 \mathrm{sec}$. The tip of the electrode ( $0.5-\mathrm{mm}$ exposed tip) was positioned $0.4 \mathrm{~mm}$ anterior, $\pm 0.25 \mathrm{~mm}$ lateral and $6.0 \mathrm{~mm}$ ventral to bregma. After surgery, mice that drank excessive amounts of water (more than $\sim 50 \mathrm{ml} /$ day) were excluded from the study. Mice that did not exhibit circadian rhythms of wheel-running in DD (for at least 10 days) were subsequently administered methamphetamine.

\section{Period analyses}

Periods were determined by $\chi^{2}$ periodogram with alpha set to 0.001 (periods ranging from 10 to $50 \mathrm{~h}$ ) of days 12-22 of methamphetamine treatment in intact and SCN-lesioned mice in DD (Clocklab). Changes in phases and/or periods of methamphetamine-induced rhythms occurred frequently, and the days used for $\chi^{2}$ periodogram analyses were altered in these instances. A minimum of 5 days was used for periodogram analyses of all mice. Methamphetamine-induced activity rhythms are characterized by period instability (especially when the SCN is disabled), so the first period that was observed after day 12 of methamphetamine treatment is reported in Table 1. Data are presented as the mean $\pm \mathrm{SD}$ period (Table 1). However, if a mouse expressed alternating short $(<24 \mathrm{~h})$ and long ( $>24 \mathrm{~h})$ 
periods, the individual values are reported in Table 2 (in this case the periods are not reported in Table 1). Mice were subsequently released into LL and periods were determined by $\chi^{2}$ periodogram of days 12-22 in LL (except where changes in the phases and/or periods occurred), with the exception of $P e r 2^{-/-}$and $\mathrm{Perl}^{-/-} / \mathrm{Per}^{-/-}$mice. Upon release into LL, $P e r 2^{-/-}$mice simultaneously exhibited two distinct rhythmic components so $\chi^{2}$ periodogram analysis was performed on days 1-35 in LL, and periodograms for each mouse are shown in the Supporting Information. After release into LL, some Per1 ${ }^{-/-} / \mathrm{Per}^{-/-}$mice sequentially expressed short and long periods so the days analyzed were altered to detect both periods (reported in Table 2). Actograms for all individual mice are shown in the Supporting Information figures and the days used for $\chi^{2}$ periodogram analysis are indicated for each mouse on these actograms. Locomotor activity was defined as arrhythmic if no significant period was detected by $\chi^{2}$ periodogram analysis.

\section{Statistical analyses}

The pooled variance method was used to define the 95\% confidence interval ( $t$-distribution) of the difference between untreated and methamphetamine-treated intact wild-type, $\mathrm{Perl}^{-/-}$, $P e r 2^{-/-}$and $P e r 3^{-/-}$mice, respectively. The periods of individual methamphetaminetreated wild-type and Per-mutant mice (intact and SCN-lesioned) were compared to the $95 \%$ confidence interval ( $t$-distribution) of the period of $\mathrm{Per}^{-/-} / \mathrm{Per}^{-/-} / \mathrm{Per} 3^{-/-}$mice. In addition, the minimum period value in each group (methamphetamine-treated, intact or SCN-lesioned, wild-type and Per-mutant mice) was used to determine the number of SDs between the period of that group and the period of methamphetamine-treated $\mathrm{Perl}^{-/-} /$ Per2 $2^{-/-} /$Per $^{-/-}$mice.

\section{Results}

\section{Methamphetamine-induced locomotor activity rhythms in Period-mutant mice in DD}

As Per $^{-/-} /$Per $^{-/-} /$Per $^{-/-}$mice had a MASCO period of $\sim 21 \mathrm{~h}$ (Pendergast et al., 2012) we examined MASCO rhythms in single Per-mutant mice to determine whether they also had short MASCO periods. We first administered methamphetamine $(0.005 \%$ in drinking water) to wild-type (n=4; Figure 1A; Supplementary Figure S1A - D), Perl ${ }^{-1-}(\mathrm{n}=6$; Figure 1C; Supplementary Figure S2A - F), Per2 ${ }^{-/-}$(n=4; Figure 1E; Supplementary Figure S3A D) and Per3 $^{-/-}(\mathrm{n}=8$; Figure 1G; Supplementary Figure S4A - H) mice in DD. During methamphetamine administration, the period of the wheel-running activity rhythm was longer than $24 \mathrm{~h}$ in wild-type, $\mathrm{Perl}^{-/-}$and $\mathrm{Per}^{-/-}$mice (Table 1; these mice have periods of $\sim 23.6 \mathrm{~h}$ without methamphetamine treatment (Pendergast et al., 2009;, 2010a)). In contrast, the period of the wheel-running rhythm was $23.5 \mathrm{~h}$ with and without methamphetamine administration in $P e r 2^{-/-}$mice (Table 1; also Pendergast et al., 2010a). The confidence intervals of the differences in periods between untreated and methamphetamine-treated wild-type [0.37, 0.84], Perl $^{-/-}[0.97,1.47]$ and Per $^{-/-}[0.56$, $0.94]$ mice were greater than (and did not overlap with) those of $P e 2^{-/-}$mice $[-0.12,0.28]$, demonstrating that intact $P e r 2^{-/}$mice did not respond to methamphetamine treatment in DD. In addition, none of the methamphetamine-treated single Per-mutant mice exhibited a rhythm with a period that was within the $95 \%$ confidence interval $[21.22,22.40]$ of the period of $\mathrm{Per}^{-/-} / \mathrm{Per}^{-/-} / \mathrm{Per}^{-/-}$mice and all mice were at least $2 \mathrm{SD}$ away from the 
$\mathrm{Per1}^{-/-} / \mathrm{Per}^{-/-} / \mathrm{Per}^{-/-}$mean period (Table S1; period values are shown for each mouse in figures in Supporting Information). These data demonstrate that no single Per gene confers a short (21-h) period in intact mice.

Because the period of the circadian activity rhythm was $23.5 \mathrm{~h}$ with and without methamphetamine treatment in $P e r 2^{-/-}$mice, we also assessed the periods of other genotypes of mice that lacked either one or both copies of functional Per2. The periods of the wheel-running rhythms were $<24 \mathrm{~h}$ with and without methamphetamine treatment in other mice lacking functional PER2 $\left(\mathrm{Perl}^{+/-} / \mathrm{Per}^{-/-} / \mathrm{Per}^{-/-}\right.$, Supplementary Figure S5; $\mathrm{Per}^{+/+} / \mathrm{Per}^{-/-} / \mathrm{Per}^{-/-}$, Supplementary Figure S6, Table 1$)$. In mice with at least a single copy of functional Per2, the periods of the wheel-running rhythms were $<23 \mathrm{~h}$ without methamphetamine administration, but $\geq 23 \mathrm{~h}$ with methamphetamine treatment $\left(\mathrm{Perl}^{-/-}\right)$ $\mathrm{Per}^{+/-} / \mathrm{Per}^{+/+}$, Supplementary Figure S7A and B; Per1 ${ }^{-/-} / \mathrm{Per}^{+/-} / \mathrm{Per}^{{ }^{-/-}}{ }^{\text {, }}$ Supplementary Figure S7C and D; Table 1).

The locomotor activity rhythm in wild-type rodents during short-term (typically $<30$ days in mice) methamphetamine treatment represents the integrated outputs of the SCN and MASCO. To eliminate the influence of the SCN on MASCO, we lesioned the SCN in wildtype ( $n=3$; Figure 1B; Supplementary Figure S1E - G), Perl-/- (n=4; Figure 1D; Supplementary Figure S2G - J), Per2 ${ }^{-/-}$(n=3; Figure 1F; Supplementary Figure S3E - G) and $\operatorname{Per}^{-/-}(\mathrm{n}=5$; Figure 1H; Supplementary Figure S4I - M) mice and investigated the methamphetamine-induced activity rhythm. The MASCO periods in wild-type, $\mathrm{Perl}^{-/-}$, $\mathrm{Per}^{-/-}$and $\mathrm{Per}^{-/-}$mice were $>25 \mathrm{~h}$ (Table 1). None of the SCN-lesioned single Permutant mice had a rhythm with a period that was within the $95 \%$ confidence interval [21.22, 22.40] of the period of $\mathrm{Per}^{-/-} / \mathrm{Per}^{-/-} / \mathrm{Per}^{-/-}$mice and all mice were at least $3 \mathrm{SD}$ away from the $\mathrm{Per}^{-/-} / \mathrm{Per}^{-/-} / \mathrm{Per}^{-/-}$mean period (Supplementary Table S1; period values are shown for each mouse in Supporting Information figures). Therefore, none of the SCNlesioned single Per-mutant mice had a short ( 21 h) MASCO period. Interestingly, one wild-type (Supplementary Figure S1E) and one $\mathrm{Perl}^{-/-}$mouse (Supplementary Figure S2I) administered methamphetamine after SCN lesion exhibited first a short $(<24 \mathrm{~h})$ and then a long (>24 h) period (Table 2).

\section{Methamphetamine-induced locomotor activity rhythms in Period-mutant mice in LL}

LL lengthens the period of the SCN-controlled activity rhythm and lowers the amplitude of the SCN oscillation in wild-type mice (Daan \& Pittendrigh, 1976; Ohta et al., 2005). Compared to wild-type mice in LL, the period of the SCN-controlled activity rhythm is longer (by $2 \mathrm{~h}$ ) in C57BL/6J Perl ${ }^{-/-}$mice, while $\mathrm{Per}^{-/-}$mice do not differ from wild types (Pendergast et al., 2010b). The period of the activity rhythm in C57BL/6J Per2-/mice is $23.5 \mathrm{~h}$ in both DD and LL (Pendergast et al., 2010b). We investigated the effect of LL on the methamphetamine-induced locomotor activity rhythms in wild-type (Figure 2A and B; Supplementary Figure S8), Perl ${ }^{-/}$(Figure 2C and D; Supplementary Figure S9), $\mathrm{Per}^{-/-}$(Figure 2E and F; Supplementary Figure S10) and $\mathrm{Per}^{-/-}$(Figure $2 \mathrm{G}$ and H; Supplementary Figure S11) mice. In LL, the periods of the methamphetamine-induced wheel-running rhythms were $29 \mathrm{~h}$ in wild-type and $P e r 3^{-/-}$mice but $35 \mathrm{~h}$ in $P e r 1^{-/-}$mice (Table 1). Upon release of $\mathrm{Per}^{-/-}$mice from DD to LL, two activity rhythms were 
simultaneously observed (Supplementary Figure S10 A-C). One activity rhythm had a short (23-h) period (similar to the period of the SCN-controlled activity rhythm in Per2 ${ }^{-/-}$mice without methamphetamine) and the other rhythm had a 30-h period (similar to the MASCO period in SCN-lesioned $\mathrm{Per}^{-/-}$mice in DD). In some Per2 ${ }^{-/-}$mice, both components persisted for the length of the recording in LL (Figure 2E) while in other mice the shortperiod component became less prominent or disappeared (Figure 2F; Supplementary Fig. S10C) and the long-period rhythm persisted. When Per2 ${ }^{-/-}$mice were maintained in 18L: $6 \mathrm{D}$ prior to release into $\mathrm{LL}$, the short $(23 \mathrm{~h})$ period was absent or disappeared quickly (Supplementary Figure S10D and E), suggesting that there may be aftereffects of entrainment to the light-dark cycle

\section{Two distinct MASCO rhythm periods in Per1 ${ }^{-/-} / \mathrm{Per}^{-/-}$mice in DD and LL}

As $\mathrm{Per}^{-/-} / \mathrm{Per}^{-/-} / \mathrm{Per}^{{ }^{-/-}}$mice had a MASCO period of $\sim 21 \mathrm{~h}$, but none of the intact and SCN-lesioned single Per-mutant mice had a period of $21 \mathrm{~h}$, we next examined methamphetamine-induced locomotor activity in $\mathrm{Perl}^{-/-} / \mathrm{Per}^{-/-}$mice in DD (Figure 3; Supplementary Fig. S12). Before methamphetamine treatment, $\mathrm{Perl}^{-/-} / \mathrm{Per}^{-/-}$mice did not exhibit circadian rhythms of wheel-running activity, demonstrating that the SCN rhythm was abrogated in DD (Figure 3). After methamphetamine administration (1-20 days), $\mathrm{Perl}^{-/-} / \mathrm{Per}^{-/-}$mice exhibited circadian rhythms of wheel-running activity. While the period of the MASCO rhythm was predominantly short, with a period of $\sim 21 \mathrm{~h}$, similar to $\mathrm{Perl}^{-/-} / \mathrm{Per}^{-/-} / \mathrm{Per}^{-/-}$mice (Supplementary Figure S13; Pendergast et al., 2012), the Perl $^{-/-} /$Per $^{-/-}$MASCO rhythm alternated between short $(\sim 21 \mathrm{~h})$ and long $(\sim 27 \mathrm{~h})$ periods of methamphetamine-induced locomotor activity (Table 2). The change in the period of the methamphetamine-induced activity rhythm always coincided with an environmental disturbance, such as changing the methamphetamine water, opening the light-tight box or changing the cage (environmental disturbances are shown on actograms in Supplementary Figure S12).

In contrast, mice with an additional single copy of $\mathrm{Per} 3\left(\mathrm{Perl}^{-/-} / \mathrm{Per}^{-/-} / \mathrm{Per}^{+/-}\right.$; Supplementary Figure S14) expressed mostly short (22 h) periods of methamphetamineinduced activity, similar to $\mathrm{Per}^{-/-} / \mathrm{Per}^{-/-} / \mathrm{Per}^{-/-}$mice (only one mouse switched from a short to along period; Supplementary Figure S13D, Table 2).

When placed in LL, some Per1 ${ }^{-/-} /$Per $^{-/-}$mice exhibited short $(\sim 17 \mathrm{~h})$ and then long ( 35 h) periods of methamphetamine-induced activity rhythms, as if the short-period component observed in DD was further shortened and the long-period component was further lengthened in LL (Figure 4A and B; Supplementary Fig. S15; Table 2). Some Perl ${ }^{-/-}$/ Per ${ }^{-/-}$mice had only one short $(\sim 17 \mathrm{~h})$ or one long $(\sim 35 \mathrm{~h})$ period activity rhythm in LL (Figure 4C-E, Supplementary Fig.S15; Table 1). Wheel-running activity was arrhythmic in $\mathrm{Perl}^{-/-} / \mathrm{Per}^{-/-}$mice maintained in LL without methamphetamine (Figure S16).

\section{The methamphetamine-induced activity rhythm in Per1 ${ }^{-/-} /$Per2 $^{-/-} /$Per $^{-/-}$mice was short in} LL

We previously demonstrated that the period of the MASCO rhythm in $\mathrm{Per}^{-/-} / \mathrm{Per}^{-/-/}$ Per $^{-/-}$mice is short ( $21 \mathrm{~h}$ ) in DD (Supplementary Figure S9; Pendergast et al., 2012). In 
LL, the period of the MASCO rhythm was $14-16 \mathrm{~h}$ in $\mathrm{Per}^{-/-} / \mathrm{Per}^{-/-} / \mathrm{Per}^{-/-}$mice (in four of five mice: Figure 5 and Supplementary Fig. S17; one mouse did not exhibit[A5] a detectable rhythm in LL; Table 1). Similar to previous studies of circadian-mutant mice (Spoelstra et al., 2002; Abraham et al., 2006), rhythms of wheel-running activity were sometimes conferred to $\mathrm{Per}^{-/-} / \mathrm{Per}^{-/-} / \mathrm{Per}^{-/-}$mice by LL even without methamphetamine treatment (Figure S18).

\section{Discussion}

\section{MASCO and the SCN are distinct circadian oscillators}

MASCO is a circadian oscillator whose output is observed in rodents during continuous consumption of low-dose methamphetamine (Honma et al., 1986; Tataroglu et al., 2006). Previous studies have demonstrated that MASCO is distinct from the SCN because (i) the methamphetamine-induced activity rhythm persists in SCN-lesioned rodents (Honma et al., 1987; Tataroglu et al., 2006) and (ii) during long-term (> 30 days) methamphetamine administration (in the light-dark cycle and in DD), MASCO de-couples from the SCN and two activity rhythms are observed, one corresponding to SCN-controlled activity and the other to MASCO-controlled activity (Honma et al., 1986; Masubuchi et al., 2000; Cuesta et al., 2011; Pendergast et al., 2012). During short-term methamphetamine administration (typically < 30 days), rodents with intact SCN express a single, free-running wheel-running activity rhythm with a period that is intermediate to the short period of the SCN rhythm and the long period of the MASCO rhythm, suggesting that the SCN and MASCO are coupled to each other (Honma et al., 1991; Masubuchi et al., 2000; Tataroglu et al., 2006). Thus, when methamphetamine is present, locomotor activity is the integrated output of the SCN and MASCO (and other oscillators) and the system is plastic over time.

\section{No single Period gene confers a short-period MASCO rhythm}

Previously we found that the MASCO rhythm in $\mathrm{Perl}^{-/-} / \mathrm{Per}^{-/-} / \mathrm{Per}^{-/-}$mice in DD (the $\mathrm{SCN}$ is disabled) is unusually short (21.5 h; Pendergast et al., 2012). In contrast, wild-type and other circadian-mutant mice have MASCO periods > $24 \mathrm{~h}$ (Tataroglu et al., 2006; Mohawk et al., 2009). To determine the contribution of each Per gene to timekeeping in MASCO, we analyzed the MASCO rhythms in single Per-mutant mice. We found that the periods of the methamphetamine-induced activity rhythms in $\mathrm{Perl}^{-/-}, \mathrm{Per}^{-/-}$and $\mathrm{Per}^{-/-}$ mice were $24 \mathrm{~h}$. None of the methamphetamine-treated single Per-mutant mice had periods approximating $21 \mathrm{~h}$. As it was possible that the 24-h MASCO periods in single Per-mutant mice could result from coupling of MASCO to the SCN, we lesioned the SCN and investigated the methamphetamine-induced rhythms. We found that the periods of MASCO in SCN-lesioned Per1 ${ }^{-/-}, \mathrm{Per}^{-/-}$and $\mathrm{Per}^{-/-}$mice were $26-30 \mathrm{~h}$ (with the exception of one SCN-lesioned wild-type and one SCN-lesioned $\mathrm{Perl}^{-/-}$mouse that displayed two sequential periods, as discussed below). SCN-lesioned wild-type mice also have MASCO periods $>24 \mathrm{~h}$ (this study and Tataroglu et al., 2006)). These data demonstrate that no single Period gene is responsible for the short-period MASCO rhythm of Per1 ${ }^{-/-} / \mathrm{Per}^{-/-} / \mathrm{Per}^{-/-}$ mice. 


\section{Two stable MASCO periods are expressed in Per1 ${ }^{-/-} /$Per2 $^{-/-}$mice}

As we found that no single Per gene is responsible for the short-period MASCO rhythm in $\mathrm{Per1}^{-/-} / \mathrm{Per}^{-/-} / \mathrm{Per}^{-/-}$mice, we next examined the MASCO rhythm in Per1 ${ }^{-/-} / \mathrm{Per}^{-/-}$ mice in DD (the SCN is disabled). We found that the MASCO rhythm alternated between a short ( $22 \mathrm{~h}$ ) and a long $(\sim 27 \mathrm{~h})$ period. The $\mathrm{Perl}^{-/-} / \mathrm{Per}^{-/-}$MASCO phenotype could result from two oscillators that are not synchronized to each other (because they have different periods and because coupling is weak as compared to wild types), resulting in relative coordination between the two. In this case, the activity rhythm would alternate, even in the absence of external stimulation. An alternative explanation for the methamphetamineinduced alternating and stable short and long periods is that the $\mathrm{PerI}^{-/-} / \mathrm{Per}^{-/-}$MASCO displays birhythmicity, which is the existence of two stable regimes of limit-cycle oscillations (Decroly \& Goldbeter, 1982; Goldbeter, 1996; Leloup \& Goldbeter, 1999). In birhythmicity, two limit cycles are simultaneously stable, so switches of the periods between two distinct values can be observed. In addition, perturbations cause the switch between the two equally stable limit cycles (it does not occur in a constant environment). In accordance with the tenets of birhythmicity, the $\mathrm{Per}^{-/-} / \mathrm{Per}^{-/-}$MASCO rhythm exhibited two fixed period values and all of the switches between the short and long period were concurrent with an environmental disturbance (most often changing the methamphetamine drinking water, but also with opening the light-tight box and changing the cage). Mohawk and colleagues found that $\mathrm{Per}^{-/-} / \mathrm{Per}^{-/-}$mice exhibit a long ( 27 h) MASCO period in DD, consistent with the long period that we observed in our experiments (Mohawk et al., 2009). It is possible that there were fewer environmental disturbances in the Mohawk et al. (2009) experiment, thus eliminating the switches between short and long periods in $\mathrm{Perl}^{-/}$/ $P e r 2^{-/}$mice. It is also possible that differences in the genetic background (i.e. generations of backcrossing to the $\mathrm{C} 57 \mathrm{BL} / 6 \mathrm{~J}$ strain) of the $\mathrm{Per}^{-/-} / \mathrm{Per}^{-/-}$mice used in this study (N15 C57BL/6J) and the mice used by Mohawk and colleagues (mixed genetic background) contributed to differences in MASCO. Computer simulations of these putative scenarios are necessary to further explore the nature of the $\mathrm{PerI}^{-/-} / \mathrm{Per}^{-/-}$MASCO.

Interestingly, when $\mathrm{Perl}^{-/-} / \mathrm{Per}^{-/-}$mice were placed in LL, some mice sequentially expressed two periods of methamphetamine-induced activity, a short $\sim 17$-h period and then a long 34-h period. In these mice, the effect of LL on the methamphetamine-induced activity rhythm may be to shorten the short period and lengthen the long period. The shortperiod MASCO rhythm in $\mathrm{Perl}^{-/-} / \mathrm{Per}^{-/-} / \mathrm{Per}^{-/-}$mice was also short $(\sim 15 \mathrm{~h})$ in LL.

Alternating short and long MASCO periods were also occasionally observed in other mice with disabled SCN (SCN-lesioned wild-type and $\mathrm{Perl}^{-/-}$mice and one $\mathrm{Perl}^{-/-} / \mathrm{Per}^{-/-}$/ $\mathrm{Per}^{-/-}$mouse). These data, in addition to the observation that phase shifts in the MASCO rhythm occur frequently in SCN-lesioned and $\mathrm{Per}^{-/-} / \mathrm{Per}^{-/-} / \mathrm{Per}^{-/-}$mice, suggest that instability may be a characteristic of MASCO. MASCO may be an inherently weak oscillator, whose instability is masked by coupling to the SCN but revealed when the SCN is disabled or its timekeeping mechanism is compromised as in $\mathrm{Per}^{-/-} / \mathrm{Per}^{-/-}$mice. 


\section{Coupling between MASCO and the SCN is altered in Per2 ${ }^{-/-}$mice}

In this study, we also sought to understand the relationship between the SCN and MASCO by analyzing wheel-running activity in intact (not SCN-lesioned) wild-type and Periodmutant mice in DD and LL. In DD, without methamphetamine, C57BL/6J wild-type and Per-mutant mice expressed similar periods of wheel-running activity ( $23.5 \mathrm{~h}$; Pendergast $e t$ al., 2009; 2010a). Upon administration of methamphetamine, the period of the activity rhythm was $24 \mathrm{~h}$ in wild-type, $\mathrm{Perl}^{-/-}$and $\mathrm{Per} 3^{-/-}$mice, suggesting that the SCN and MASCO are coupled (at least during short-term, i.e. less $<30$ days, methamphetamine treatment). In contrast, the period of the wheel-running activity rhythm in $\mathrm{Per}^{-/-}$mice was $23.5 \mathrm{~h}$ with and without methamphetamine consumption. This may occur if the $P e r 2^{-/-}$ MASCO is insensitive to methamphetamine. However, this is an unlikely explanation because the long-period MASCO rhythm (similar MASCO period as SCN-lesioned wildtypes) was clearly expressed in $P e r 2^{-/-}$mice when the $\mathrm{SCN}$ was lesioned. Thus, it is more likely that the SCN and MASCO are not coupled in $\mathrm{Per}^{-/-}$mice. Because we only observed one circadian activity rhythm component in SCN-intact $P e r 2^{-/-}$mice, we propose that MASCO output is masked or inhibited in DD. Furthermore, our data suggest that a single copy of Per2 is sufficient to restore coupling between the SCN and MASCO.

Rather than expressing a single integrated methamphetamine-induced wheel-running activity rhythm in LL, $\mathrm{Per}^{-/-}$mice initially had two free-running components, one corresponding to the period of the SCN (which is $23.5 \mathrm{~h}$ in LL in $P e r 2^{-/-}$mice; Pendergast et al., 2010b) and the other to the MASCO period. This is additional evidence that the SCN and MASCO are not coupled in $\mathrm{Per}^{-/-}$mice. However, unlike in DD, MASCO output is not masked in LL (and thus we observe both the SCN- and MASCO-controlled activity rhythms). With extended exposure to LL, the short-period component became less visible and disappeared in some mice; this may correspond to the gradual effect of LL to desynchronize SCN neurons (Ohta et al., 2005). It is possible that, as the amplitude of the SCN oscillator decreases, MASCO gradually suppresses SCN output.

Similar to our results in Per2 ${ }^{-/-}$mice, Tataroglu et al. (2006) reported that methamphetamine treatment did not affect the period the locomotor activity rhythm in intact $\mathrm{C} 3 \mathrm{H}$ mice in DD. In contrast, the MASCO period in SCN-lesioned $\mathrm{C} 3 \mathrm{H}$ mice was $\sim 24.5 \mathrm{~h}$, while the MASCO period in SCN-lesioned $\mathrm{Per}^{-/-}$mice was $\sim 27 \mathrm{~h}$, and $\mathrm{C} 3 \mathrm{H}$ mice express only one activity component during methamphetamine treatment in LL. Therefore, unlike $\mathrm{Per}^{-/-}$mice, coupling between the $\mathrm{SCN}$ and MASCO is probably intact in $\mathrm{C} 3 \mathrm{H}$ mice, but the period of MASCO may be shortened in $\mathrm{C} 3 \mathrm{H}$ mice compared to $\mathrm{C} 57 \mathrm{BL} / 6 \mathrm{~J}$ mice.

\section{Working model of the in vivo relationship between MASCO and the SCN}

Based on our experiments, we propose a working model of the SCN-MASCO integrated circuit in wild-type mice (Figure 6). In our model, the SCN and MASCO are mutually coupled circadian oscillators that also inhibit each other's output, but the strengths of these interactions are modulated by environmental factors. The effector, which is an unidentified neural circuit or locus, integrates the output from the MASCO and SCN and drives wheelrunning activity. Wheel-running activity itself may be critical for coupling between MASCO and the $\mathrm{SCN}$ as it was previously demonstrated that the period of the locomotor activity 
rhythm was not lengthened in rats administered methamphetamine without a running wheel (Honma et al., 1991). Future studies should examine the role of wheel-running activity in MASCO-SCN coupling.

In DD, the SCN is a robust circadian oscillator that is coupled to MASCO and also suppresses its output (Figure 6A). We propose that, in the absence of methamphetamine, MASCO is a weak oscillator so it has little influence on the SCN and it does not appreciably suppress the output of the SCN. Thus, in DD without methamphetamine, the locomotor activity rhythm is largely (or completely) controlled by the SCN. Upon addition of methamphetamine, MASCO becomes a robust circadian oscillator, thus increasing its coupling to the SCN and its suppression of SCN output (Figure 6B). The resulting locomotor activity rhythm is an integration of both SCN and MASCO outputs. During longterm methamphetamine administration (> 30 days), the SCN and MASCO become decoupled and two activity rhythms are expressed. In our model, we assume that methamphetamine does not affect the period of the SCN; we thus conceptualize that the $\mathrm{SCN}$ and MASCO become decoupled rather than exhibit relative coordination. However, our interpretation is limited as it is possible that methamphetamine affects the period of the SCN. Unfortunately, the effect of methamphetamine on the period of the SCN cannot be determined as this would require methamphetamine treatment of mice with MASCO lesions and we do not know the anatomical locus of the MASCO.

In LL, without methamphetamine, both the SCN and MASCO are weak oscillators (Figure 6C). Upon addition of methamphetamine, MASCO becomes a robust oscillator, increasing its coupling to the SCN and its suppression of SCN output (we assume that LL does not affect the amplitude of MASCO; Figure 6D). As we assume that DD and LL do not differentially affect MASCO, we propose that the period-lengthening of the methamphetamine-induced activity rhythm in LL is largely attributed to the effects of light on the SCN. This is consistent with our data showing that the magnitude of lengthening of the methamphetamine-induced rhythm in $\mathrm{Perl}^{-/-}$mice is greater than the lengthening in wild-type and $P e r 3^{-/-}$mice in LL (which mirrors the respective effects of LL on the SCNcontrolled activity rhythms in these mice; Pendergast et al., 2010b). However, we cannot rule out the possibility that light acts directly on MASCO.

$\mathrm{Per}^{-/-}$mice are unique in that their SCN and MASCO are probably not coupled (even during short-term methamphetamine administration). Because we found that $P e r 2^{-/}$mice exhibit one component (corresponding to the period of the $\mathrm{SCN}$ ) in DD but two components in LL, we propose that the SCN suppresses the output of MASCO in DD but not in LL. We attribute the strength of the suppression to the robustness of the SCN oscillator. In DD, the $\mathrm{Per}^{-/-} \mathrm{SCN}$ is a robust oscillator that is not coupled to MASCO, but suppresses MASCO output. However, in LL the Per2 ${ }^{-/-} \mathrm{SCN}$ is a weak oscillator that is not coupled to MASCO and does not suppress MASCO output, so two components are observed upon release into LL. The function of PER2 in SCN-MASCO coupling is unknown and should be investigated in future studies.

This study demonstrates the complexity of the multi-oscillator circadian system and highlights the fact that the output measured from the animal represents the integration of 
multiple oscillators. Computational modeling is critical for further understanding the complex relationships between these oscillators.

\section{Supplementary Material}

Refer to Web version on PubMed Central for supplementary material.

\section{Acknowledgments}

This research was supported by a National Science Foundation grant IOS-1146908 to S.Y. K.D.N. was supported by resources of the Tennessee Valley Healthcare System and National Institutes of Health grants, DK085712 and the Diabetes Research and Training Center DK020593. The Vanderbilt University Department of Biostatistics Basic Science Clinic provided guidance regarding statistical analyses of data. We thank Dr Gisele A. Oda and Dr Gen Kurosawa for insightful discussion of the data. We also thank Dr Wataru Nakamura for technical advice in performing SCN lesion surgery.

\section{Abbreviations}

DD constant darkness

LL constant light

MASCO methamphetamine-sensitive circadian oscillator

Per Period

SCN suprachiasmatic nucleus

\section{References}

Abraham D, Dallmann R, Steinlechner S, Albrecht U, Eichele G, Oster H. Restoration of circadian rhythmicity in circadian clock-deficient mice in constant light. J Biol Rhythms. 2006; 21:169-176. [PubMed: 16731656]

Bae K, Jin X, Maywood ES, Hastings MH, Reppert SM, Weaver DR. Differential functions of mPer1, mPer2, and mPer3 in the SCN circadian clock. Neuron. 2001; 30:525-536. [PubMed: 11395012]

Cuesta M, Aungier J, Morton AJ. The methamphetamine-sensitive circadian oscillator is dysfunctional in a transgenic mouse model of Huntington's disease. Neurobiol Dis. 2011; 45:145-155. [PubMed: 21820053]

Daan S, Pittendrigh CS. A Functional Analysis of Circadian Oscillators in Nocturnal Rodents: III. Heavy Water and Constant Light: Homeostasis of Frequency? J. Comp. Physiol. 1976; 106:253266.

Davidson AJ. Lesion studies targeting food-anticipatory activity. Eur J Neurosci. 2009; 30:1658-1664. [PubMed: 19863659]

Decroly O, Goldbeter A. Birhythmicity, chaos, and other patterns of temporal self-organization in a multiply regulated biochemical system. Proc Natl Acad Sci U S A. 1982; 79:6917-6921. [PubMed: 6960354]

Goldbeter, A. Biochemical oscillations and cellular rhythms : the molecular bases of periodic and chaotic behaviour. New York: Cambridge University Press, Cambridge; 1996.

Honma K, Honma S, Hiroshige T. Disorganization of the rat activity rhythm by chronic treatment with methamphetamine. Physiol Behav. 1986; 38:687-695. [PubMed: 3823184]

Honma K, Honma S, Hiroshige T. Activity rhythms in the circadian domain appear in suprachiasmatic nuclei lesioned rats given methamphetamine. Physiol Behav. 1987; 40:767-774. [PubMed: 3313452]

Honma S, Honma K, Hiroshige T. Methamphetamine effects on rat circadian clock depend on actograph. Physiol Behav. 1991; 49:787-795. [PubMed: 1881985] 
Honma S, Yasuda T, Yasui A, van der Horst GT, Honma K. Circadian behavioral rhythms in Cry1/ Cry2 double-deficient mice induced by methamphetamine. J Biol Rhythms. 2008; 23:91-94. [PubMed: 18258761]

Leloup JC, Goldbeter A. Chaos and birhythmicity in a model for circadian oscillations of the PER and TIM proteins in drosophila. J Theor Biol. 1999; 198:445-459. [PubMed: 10366496]

Masubuchi S, Honma S, Abe H, Ishizaki K, Namihira M, Ikeda M, Honma K. Clock genes outside the suprachiasmatic nucleus involved in manifestation of locomotor activity rhythm in rats. Eur $\mathbf{J}$ Neurosci. 2000; 12:4206-4214. [PubMed: 11122332]

Mistlberger RE. Circadian food-anticipatory activity: formal models and physiological mechanisms. Neurosci Biobehav Rev. 1994; 18:171-195. [PubMed: 8058212]

Mohawk JA, Baer ML, Menaker M. The methamphetamine-sensitive circadian oscillator does not employ canonical clock genes. Proc Natl Acad Sci U S A. 2009; 106:3519-3524. [PubMed: 19204282]

Ohta H, Yamazaki S, McMahon DG. Constant light desynchronizes mammalian clock neurons. Nat Neurosci. 2005; 8:267-269. [PubMed: 15746913]

Pendergast JS, Friday RC, Yamazaki S. Endogenous rhythms in period1 mutant suprachiasmatic nuclei in vitro do not represent circadian behavior. J Neurosci. 2009; 29:14681-14686. [PubMed: 19923301]

Pendergast JS, Friday RC, Yamazaki S. Distinct functions of Period 2 and Period3 in the mouse circadian system revealed by in vitro analysis. PLoS One. 2010a; 5:e8552. [PubMed: 20072700]

Pendergast JS, Friday RC, Yamazaki S. Photic Entrainment of Period Mutant Mice is Predicted from Their Phase Response Curves. J Neurosci. 2010b; 30:12179-12184. [PubMed: 20826680]

Pendergast JS, Oda GA, Niswender KD, Yamazaki S. Period determination in the food-entrainable and methamphetamine-sensitive circadian oscillator(s). Proc Natl Acad Sci U S A. 2012; 109:1421814223. [PubMed: 22891330]

Pezuk P, Mohawk JA, Yoshikawa T, Sellix MT, Menaker M. Circadian organization is governed by extra-SCN pacemakers. J Biol Rhythms. 2010; 25:432-441. [PubMed: 21135159]

Shearman LP, Jin X, Lee C, Reppert SM, Weaver DR. Targeted disruption of the mPer3 gene: subtle effects on circadian clock function. Mol Cell Biol. 2000; 20:6269-6275. [PubMed: 10938103]

Spoelstra K, Oklejewicz M, Daan S. Restoration of self-sustained circadian rhythmicity by the mutant clock allele in mice in constant illumination. J Biol Rhythms. 2002; 17:520-525. [PubMed: 12465885]

Takahashi, JS.; Turek, FW.; Moore, RY. Circadian clocks. New York: Kluwer Academic/Plenum Publishers; 2001.

Tataroglu O, Davidson AJ, Benvenuto LJ, Menaker M. The methamphetamine-sensitive circadian oscillator (MASCO) in mice. J Biol Rhythms. 2006; 21:185-194. [PubMed: 16731658]

Yamazaki S, Numano R, Abe M, Hida A, Takahashi R, Ueda M, Block GD, Sakaki Y, Menaker M, Tei H. Resetting central and peripheral circadian oscillators in transgenic rats. Science. 2000; 288:682-685. [PubMed: 10784453]

Yoo SH, Yamazaki S, Lowrey PL, Shimomura K, Ko CH, Buhr ED, Siepka SM, Hong HK, Oh WJ, Yoo OJ, Menaker M, Takahashi JS. PERIOD2::LUCIFERASE real-time reporting of circadian dynamics reveals persistent circadian oscillations in mouse peripheral tissues. Proc Natl Acad Sci U S A. 2004; 101:5339-5346. [PubMed: 14963227] 

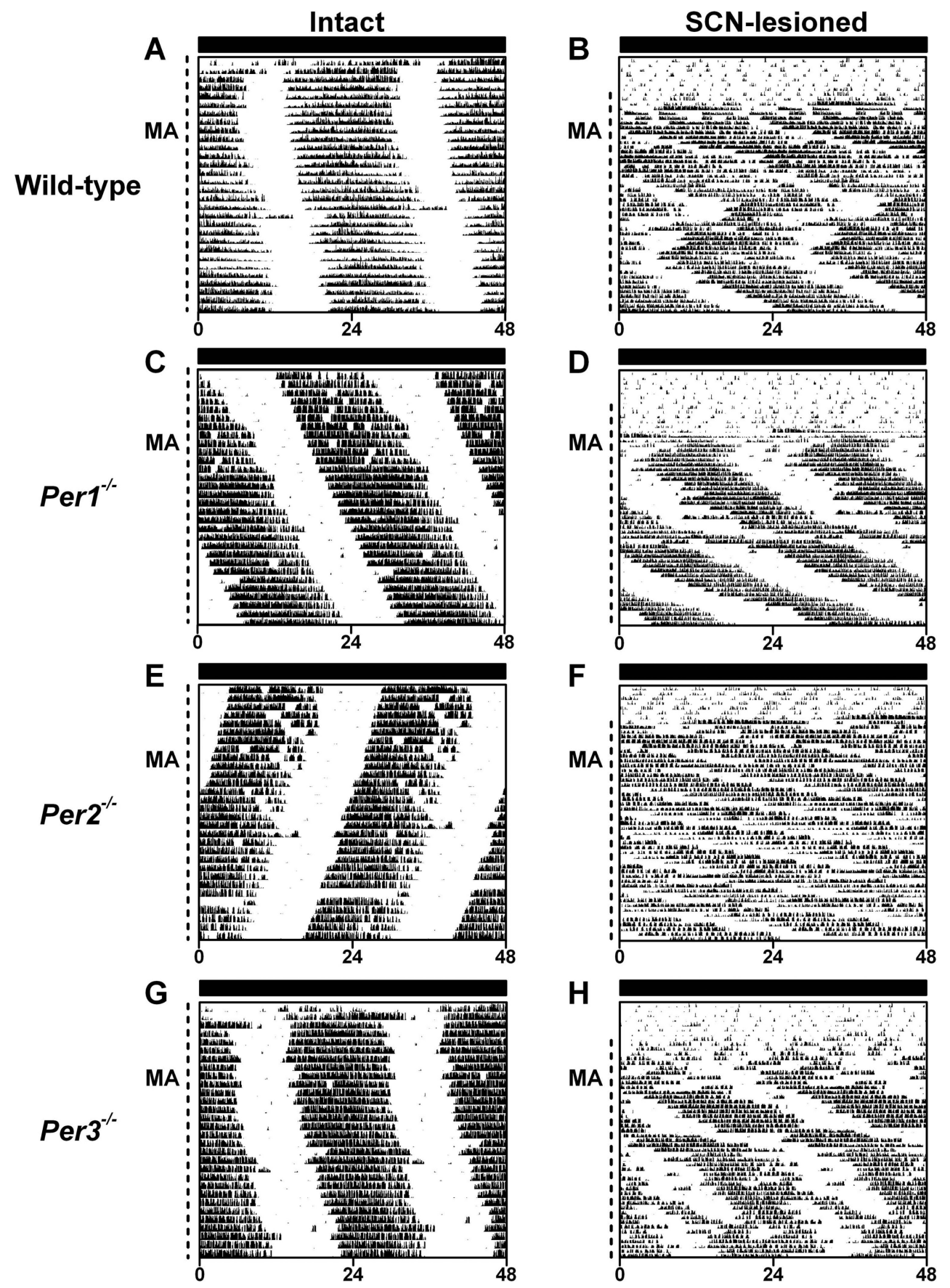

Figure 1.

Methamphetamine-induced activity rhythms in Period-mutant mice in DD. Representative double-plotted actograms (5-min bins) of wheel-running activity recorded from (A and $\mathrm{B}$ ) wild-type, (C and D) Per1 ${ }^{-/-}$, (E and F) Per2 ${ }^{-/-}$and (G and H) Per3 ${ }^{-/-}$mice ( $x$-axis, time in hours; $y$-axis, days). (A, C, E and G) Intact mice were maintained in DD (black bars above actograms) and administered $0.005 \%$ methamphetamine (MA) in their drinking water (days 1-29 of MA treatment are shown in the actograms). (B, D, F and H) Mice were SCNlesioned and maintained in DD (without MA) to verify the absence of circadian activity 
rhythms (7 days shown in actograms). SCN-lesioned mice were then administered $0.005 \%$ MA in their drinking water (45 days of MA treatment are shown in the actograms in B, D, F and $\mathrm{H})$. The time of MA treatment is indicated by dotted lines to the left of the actograms. 

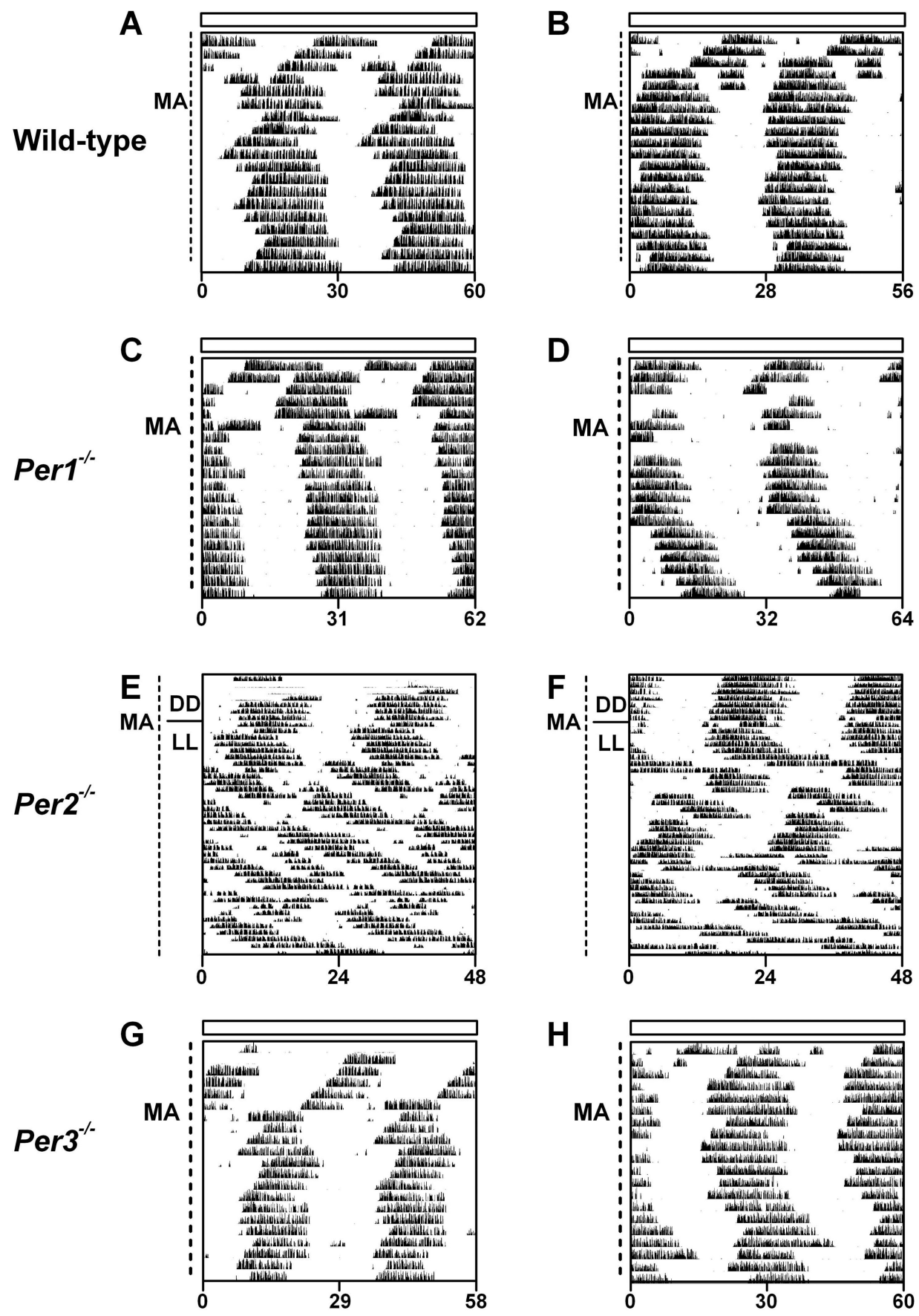

Figure 2.

Methamphetamine-induced activity rhythms in Period-mutant mice in LL. Representative double-plotted actograms (5-min bins; $x$-axis, time in hours; $y$-axis, cycles) of wheel-running activity recorded from (A and B) wild-type, (C and D) PerI ${ }^{-/-}$and (G and H) Per3 ${ }^{-/-}$mice maintained in LL (200-300 lux; white bars above actograms) and administered $0.005 \%$ methamphetamine (MA) in their drinking water. (E and F) $\mathrm{Per}^{-/-}$mice were administered $0.005 \%$ MA and maintained in DD for 7 days and then released into LL. The time of MA administration is indicated by dotted lines to the left of the actograms. The data are plotted 
on (A and H) 30-h, (B) 28-h, (C) 31-h, (D) 32-h, (E and F) 24-h and (G) 29-h cycles. All mice were previously maintained in DD and then released into LL (activity in DD shown only for $\mathrm{Per}^{-/-}$mice to aid in the visualization of the two components in LL). 

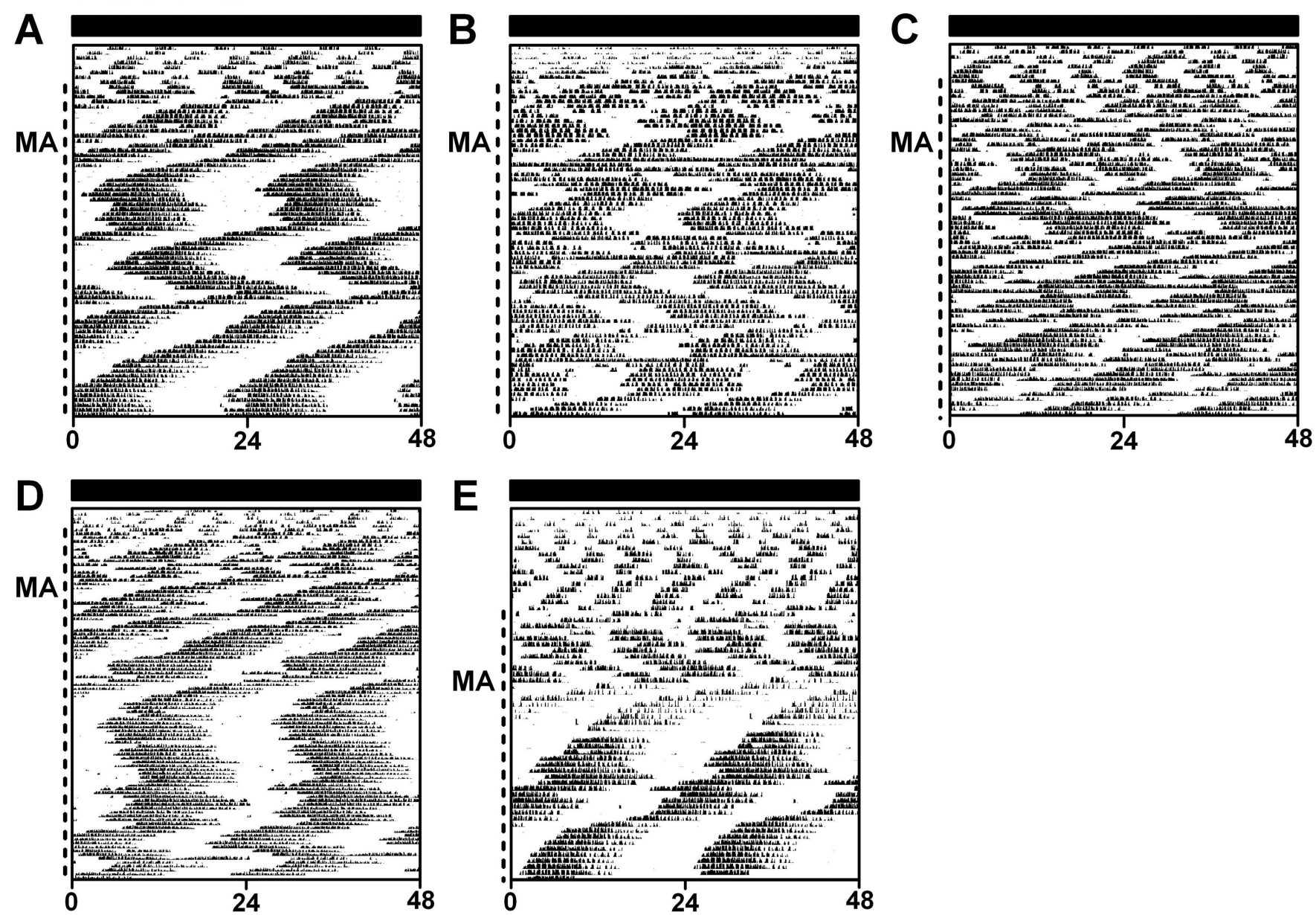

Figure 3.

Two stable MASCO periods were expressed in $\mathrm{Perl}^{-/-} / \mathrm{Per}^{-/-}$mice in DD. Doubleplotted actograms (5-min bins; $x$-axis, time in hours; $y$-axis, days) of wheel-running activity recorded from $\mathrm{Per}^{-/} / \mathrm{Per}^{-/-}$mice maintained in DD (indicated by black bars above actograms). Six to eight days of activity prior to methamphetamine (MA) administration are shown. Mice were then administered $0.005 \% \mathrm{MA}$ in their drinking water. The time of MA administration is indicated by dotted lines to the left of the actograms. All individual actograms are shown $(n=5)$. 

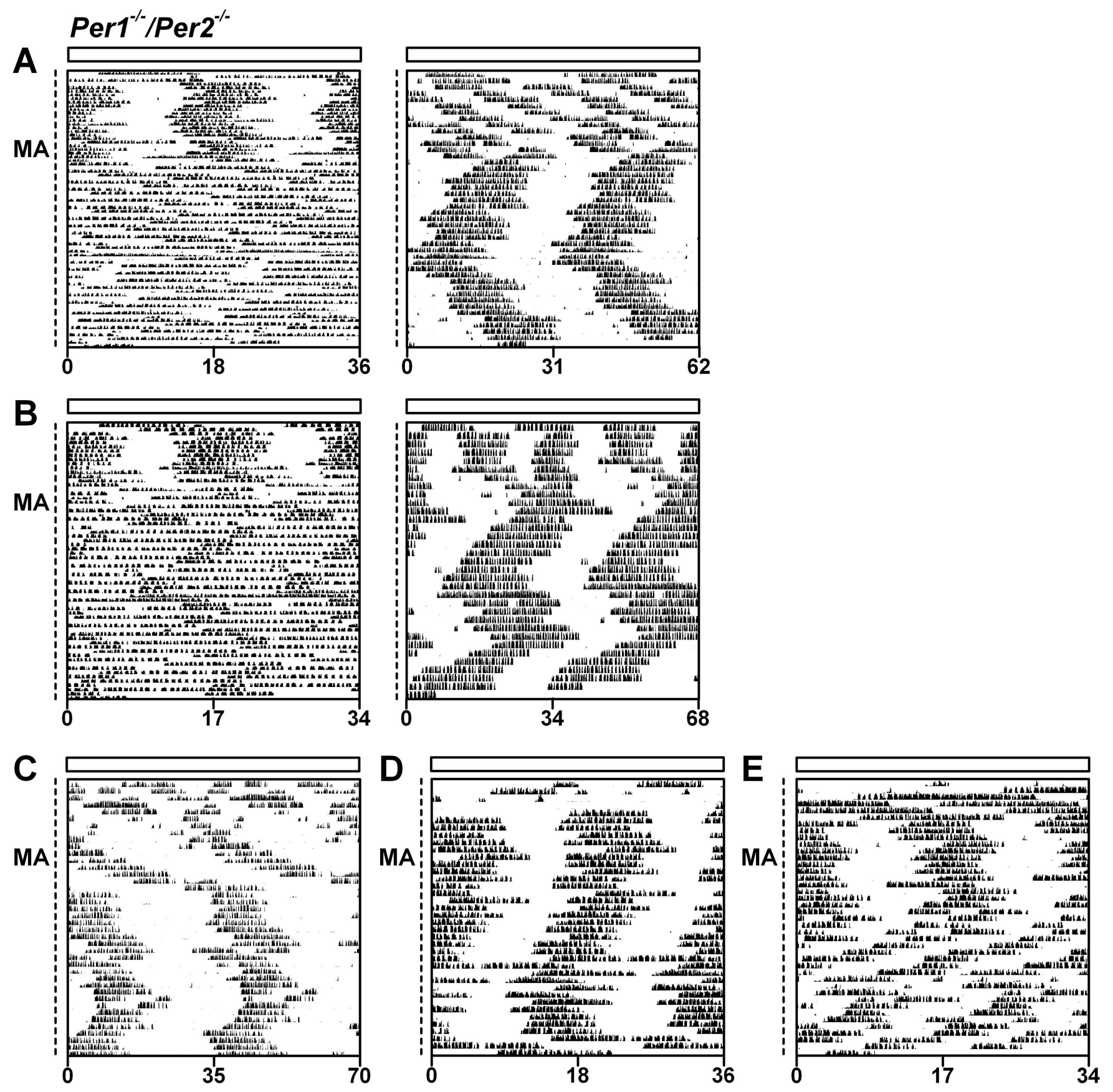

Figure 4.

Methamphetamine-induced activity rhythms in $\mathrm{Per}^{-/-} / \mathrm{Per}^{-/-}$mice in LL. Double-plotted actograms (5-min bins; $x$-axis, time in hours; $y$-axis, cycles) of wheel-running activity recorded from $\mathrm{Perl}^{-/-} / \mathrm{Per}^{-/-}$mice maintained in LL (indicated by white bars above actograms) and administered $0.005 \%$ methamphetamine (MA) in their drinking water. The mice were previously maintained in DD with methamphetamine (shown in Figure 3) and then released into LL. Data from all individual mice are shown $(n=5)$. The mice in (A) and (B) first displayed a short and then a long period, so two actograms are shown for each of these mice. The data for the mouse shown in A are plotted on an 18-h cycle (left panel) and 
a 31-h cycle (right panel). The data for the mouse shown in B are plotted in a 17-h cycle (left panel) and a 34-h cycle (right panel). The mice shown in C-E only displayed a single period and are plotted on 35-h, 18-h and 17-h cycles, respectively. 


\section{$\operatorname{Per1}^{-/-} / \operatorname{Per}^{-/-} /$Per3 $^{-/-}$}
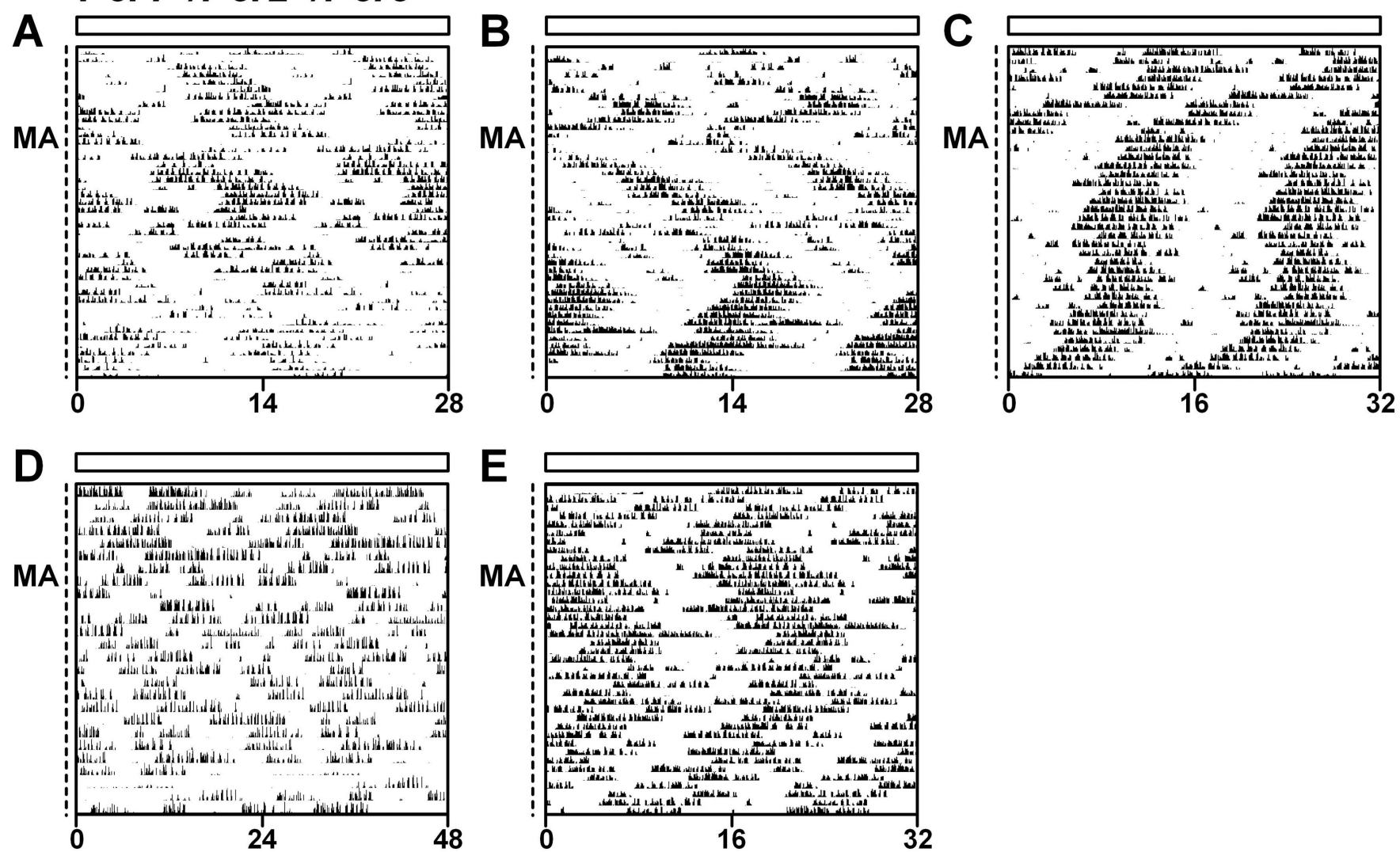

Figure 5.

MASCO rhythms in $\mathrm{Per1}^{-/-} / \mathrm{Per}^{-/-} / \mathrm{Per}^{-/-}$mice were shortened in LL. Double-plotted actograms (5-min bins; $x$-axis, time in hours; $y$-axis, cycles) of wheel-running activity recorded from $\mathrm{Per}^{-/-} / \mathrm{Per}^{-/-} / \mathrm{Per}^{-/-}$mice maintained in LL (indicated by white bars above actograms) and administered $0.005 \%$ methamphetamine (MA) in their drinking water. The mice were previously maintained in DD with methamphetamine and then released into LL. Data from all individual mice in LL are shown $(\mathrm{n}=5)$. The data are plotted on (A and $\mathrm{B})$ 14-h, (C and E) 16-h and (D) 24-h cycles. No significant period was detected in the mouse shown in D. 
A Constant darkness: No MA

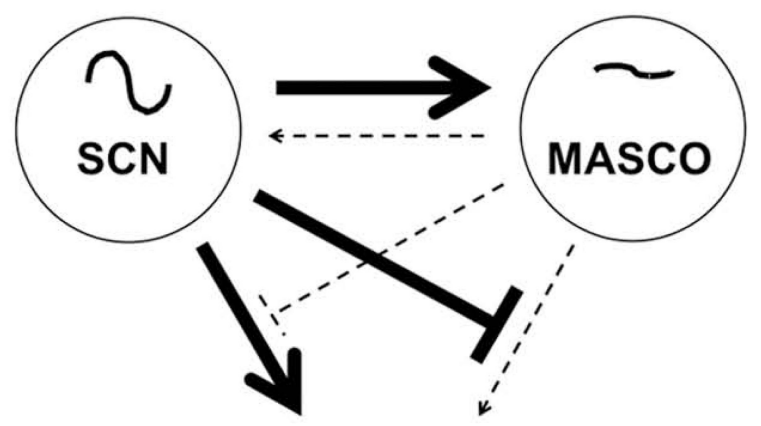

Effector

C
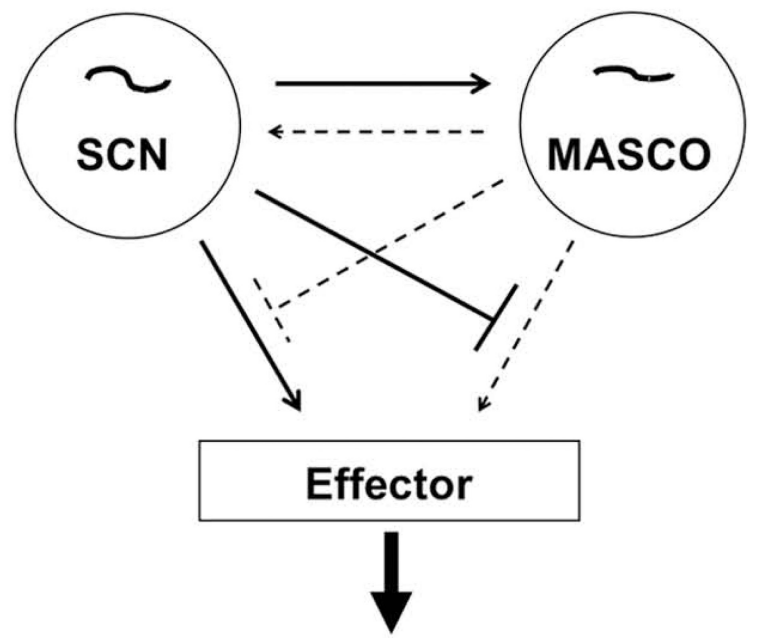

Locomotor activity rhythm
B Constant darkness: + MA

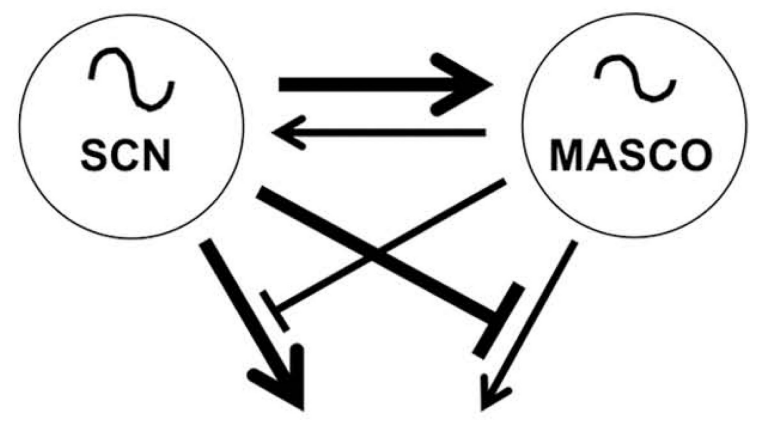

Effector

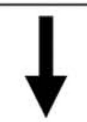

Locomotor activity rhythm

D Constant light: + MA
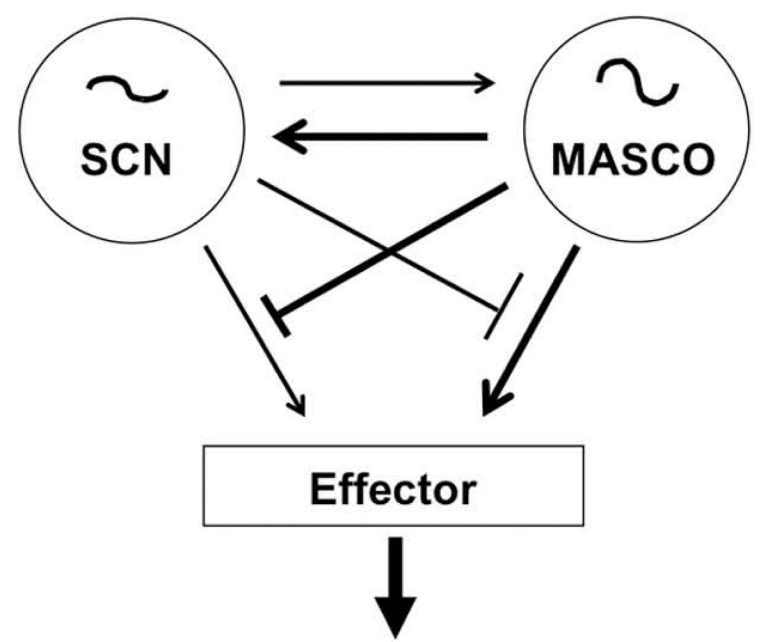

Locomotor activity rhythm

Figure 6.

Working model of the in vivo relationship between MASCO and the SCN. (A and B) In DD, the $\mathrm{SCN}$ is a robust circadian oscillator that is coupled to MASCO and also inhibits its output. (A and C) We propose that, in the absence of methamphetamine, MASCO is a weak oscillator so it has little influence on the SCN and it does not appreciably inhibit the output of the SCN. Thus, in (A) DD without methamphetamine, the locomotor activity rhythm is largely (or completely) controlled by the SCN. (B) Upon addition of methamphetamine in $\mathrm{DD}, \mathrm{MASCO}$ becomes a robust circadian oscillator, thus increasing its coupling to the SCN 
and its inhibition of SCN output. During long-term methamphetamine administration, the SCN and MASCO become decoupled and two activity rhythms are expressed (not shown). $\mathrm{Per}^{-/-}$mice are unique in that their SCN and MASCO are probably not coupled even during short-term methamphetamine administration (not shown). (C) In LL, without methamphetamine, both the SCN and MASCO are weak oscillators. (D) Upon addition of methamphetamine, MASCO becomes a robust oscillator, increasing its coupling to the SCN and its inhibition of SCN output (assuming that LL does not affect the amplitude of MASCO). 


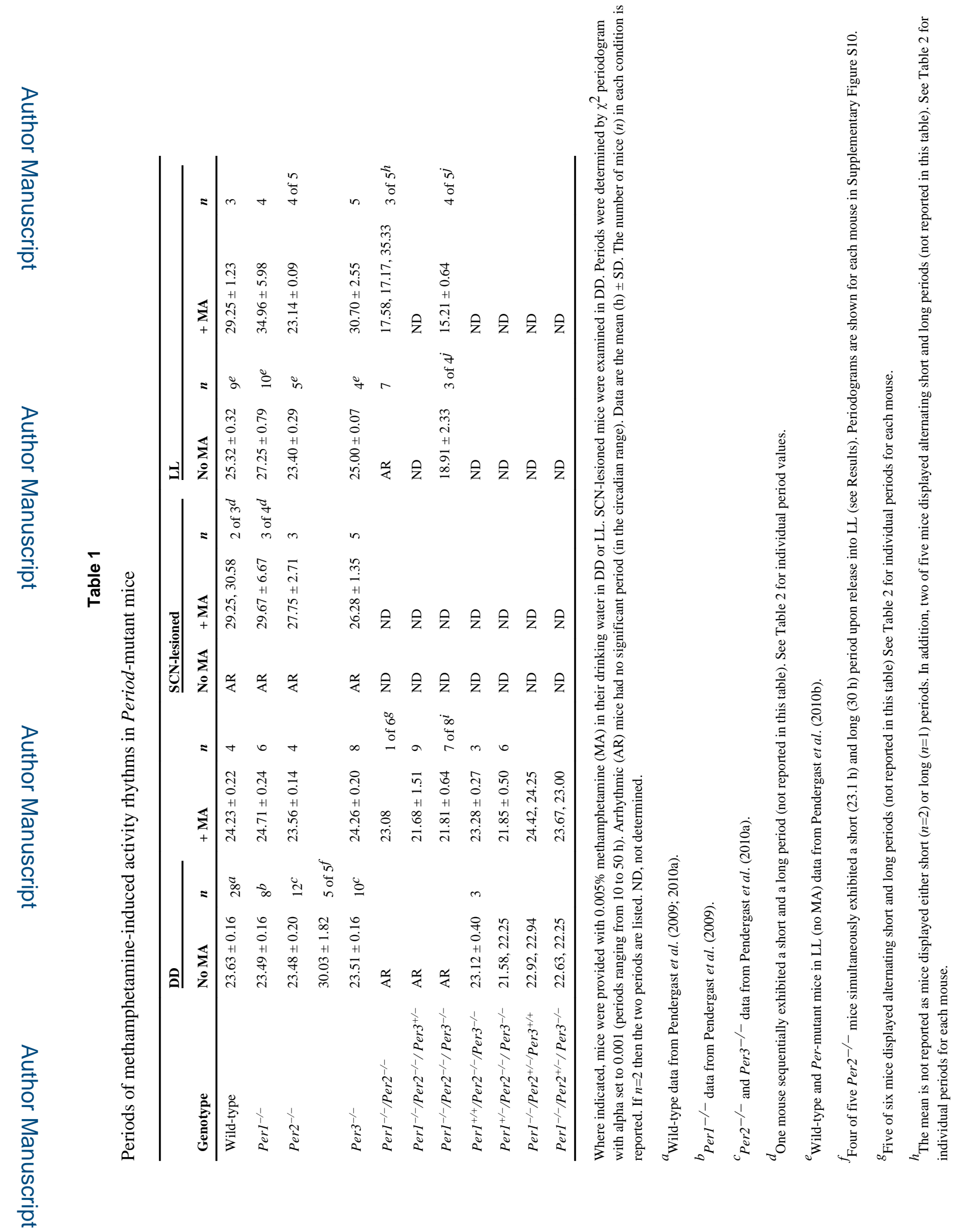

Eur J Neurosci. Author manuscript; available in PMC 2015 January 22. 


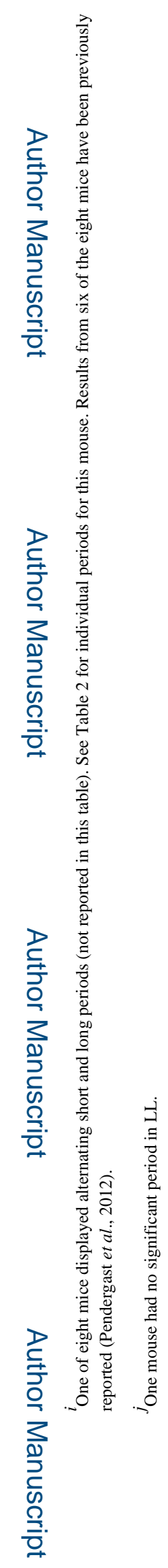

Eur J Neurosci. Author manuscript; available in PMC 2015 January 22. 


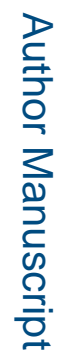

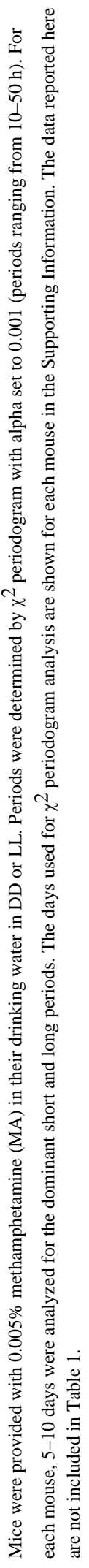

